\title{
Existence of the classical and strong solutions for fractional semilinear initial value problems
}

\author{
A. Al-Omari ${ }^{1}$ and H. Al-Saadi ${ }^{2 *}$
}

\section{"Correspondence:}

hasa112@hotmail.com

${ }^{2}$ Mathematics Department, Faculty

of Applied Sciences, Umm Al-Qura

University, Makkah, Saudi Arabia

Full list of author information is

available at the end of the article

\begin{abstract}
We prove the existence and uniqueness of classical and strong solutions of a fractional semilinear evolution equation using the method of a semigroup and the Banach fixed theorem.
\end{abstract}

MSC: $54 \mathrm{H} 25 ; 47 \mathrm{H} 10 ; 34 \mathrm{~A} 12 ; 34 \mathrm{~A} 40 ; 34 \mathrm{k} 5$

Keywords: Fixed point theorems; Existence; Classical solution; Strong solution; Fractional differential equations

\section{Introduction}

Fractional differential equations are important mathematical models of some practical problems in many fields such as polymer rheology, chemistry physics, heat conduction, fluid flows, electrical networks, and many other branches of science (see [1, 2, 12, 14]). Consequently, the fractional calculus and its applications in various fields of science and engineering have received much attention, and many papers and books on fractional calculus, fractional differential equations have appeared (see $[2,4,10])$. It should be noted that the theory of nonlinear fractional differential equation boundary value problems receives more and more attention (see [1, 3, 13]). There has been significant development in fractional differential equations in recent years; see the monographs of Lakshmikantham et al. [8], Kilbas et al. [7], Podlubny [11], Miller and Ross [9].

Definition 1.1 ([10]) Let $X$ be a Banach space. A one parameter $T(t), 0 \leq t<\infty$, be a bounded linear operators from $X$ into $X$ is a semigroup of a bounded linear operator on $X$ if

(1) $T(0)=I(I$ is the identity operator on $X)$.

(2) $T(t+s)=T(t) T(s)$ for every $t, s \geq 0$ (the semigroup property).

A semigroup of bounded linear operators, $T(t)$, is uniformly continuous if

$$
\lim _{t \rightarrow 0}\|T(t)-I\|=0
$$


The linear operator $A$ defined by

$$
D(A)=\left\{x \in X: \lim _{t \rightarrow 0} \frac{T(t) x-x}{t} \text { exists }\right\}
$$

and

$$
A x=\lim _{t \rightarrow 0} \frac{T(t) x-x}{t}=\left.\frac{d^{+} T(t) x}{d t}\right|_{t=0} \text { for } x \in D(A)
$$

is the infinitesimal generator of the semigroup $T(t), D(A)$ is the domain of $A$.

Jardat et al. [6] considered the existence and uniqueness of mild solution for the semilinear initial value problem of non-integer order.

Zhou and Jiao [14] investigated the existence and uniqueness of mild solutions of the nonlocal Cauchy problem with fractional evolution equations in an arbitrary Banach space.

In this paper we study the existence and uniqueness of the classical and strong solutions for the nonlinear evolution equation

$$
\begin{aligned}
& \left.\left.u^{(\alpha)}(t)=A u(t)+f(t, u(t), G u(t), S u(t)), \quad t_{0}<t<T ; \alpha \in\right] 0,1\right], \\
& u\left(t_{0}\right)=u_{0},
\end{aligned}
$$

where $\mathrm{A}$ is the infinitesimal generator of a $C_{0}$-semigroup $\{T(t) ; t \geq 0\}$ on a Banach space $\mathrm{X}$ and $f:\left[t_{0}, T\right] \times X \times X \times X \rightarrow X$ is continuous in $t$, and

$$
\begin{aligned}
& G u(t)=\int_{t_{0}}^{t} K(t, s) u(s) d s, \quad K \in C\left[D, R^{+}\right], \\
& S u(t)=\int_{t_{0}}^{t} H(t, s) u(s) d s, \quad H \in C\left[D_{0}, R^{+}\right],
\end{aligned}
$$

where $D=\left\{(t, s) \in R^{2}: t_{0} \leq s \leq t \leq T\right\}, D_{0}=\left\{(t, s) \in R^{2}: t_{0} \leq t, s \leq T\right\}$.

Then we prove that the existence and uniqueness of classical and strong solutions if $f$ satisfies certain conditions.

Definition 1.2 ([1]) The fractional (arbitrary) order integral of the function $h \in L^{1}([a, b]$, $\left.\mathbb{R}^{+}\right)$of order $\alpha \in \mathbb{R}^{+}$is defined by

$$
I_{a}^{\alpha} h(t)=\int_{a}^{t} \frac{(t-s)^{\alpha-1}}{\Gamma(\alpha)} h(s) d s,
$$

where $\Gamma$ is the gamma function. When $a=0$, we write $I^{\alpha} h(t)=h(t) * \varphi_{\alpha}(t)$, where $\varphi_{\alpha}(t)=$ $\frac{t^{\alpha-1}}{\Gamma(\alpha)}$ for $t>0$, and $\varphi_{\alpha}(t)=0$ for $t \leq 0$, and $\varphi_{\alpha} \rightarrow \delta(t)$ as $\alpha \rightarrow 0$, where $\delta$ is the delta function.

Definition 1.3 ([1]) For a function $h$ given on the interval $[a, b]$, the Caputo fractional order derivative of $h$, is defined by,

$$
\left({ }^{c} D_{a^{+}}^{\alpha} h\right)(t)=\frac{1}{\Gamma(n-\alpha)} \int_{a}^{t}(t-s)^{n-\alpha-1} h^{(n)}(s) d s
$$

Here $n=[\alpha]+1$ and $[\alpha]$ denotes the integer part of $\alpha$. 


\section{Main results}

In this section, we shall introduce some basic definitions, notations and lemmas which are used throughout this paper.

Definition 2.1 A function $u:\left[t_{0}, T[\rightarrow X\right.$ is a classical solution of the initial value problem (1.1) on $\left[t_{0}, T\left[\right.\right.$ if $u$ is continuous on $\left[t_{0}, T\right.$, continuously differentiable of order $\alpha$ on $] t_{0}, T$, $u \in D(A)$ for $t \in] t_{0}, T$ [ and the initial value problem (1.1) is satisfied on $\left[t_{0}, T[\right.$.

Let $T(t)$ be the $C_{0}$-semigroup generator by $A$ and let $u$ be a solution of Eq. (1.1). Then the valued function $g(s)=T(t-s) u(s)$ is differentiable of order $\alpha$ for $t_{0}<s<t$ and

$$
\begin{aligned}
g^{(\alpha)}(s) & =T(t-s) u^{(\alpha)}(s)-u(s) A^{\alpha} T(t-s) \\
& =A T(t-s) u(s)+T(t-s) f(s, u(s), G u(s), S u(s))-u(s) A^{\alpha} T(t-s) \\
& =T(t-s) f(s, u(s), G u(s), S u(s))-u(s)\left(A-A^{\alpha}\right) T(t-s) .
\end{aligned}
$$

If $f \in L^{1}\left(\left[t_{0} ; T\right]: X\right)$ then $T(t-s) f(s, u(s), G u(s), S u(s))-u(s)\left(A-A^{\alpha}\right) T(t-s)$ is integrable of order $\alpha$ and integrating from $t_{0}$ to $t$ yields

$$
\begin{gathered}
\left.T(t-s) u(s)\right|_{t_{0}} ^{t}=\frac{1}{\Gamma(\alpha)} \int_{t_{0}}^{t}(t-s)^{\alpha-1} T(t-s) f(s, u(s), G u(s), S u(s)) d s \\
+\frac{1}{\Gamma(\alpha)} \int_{t_{0}}^{t}(t-s)^{\alpha-1} T(t-s)\left(A-A^{\alpha}\right) u(s) d s, \\
u(t)=T\left(t-t_{0}\right) u_{0}+\frac{1}{\Gamma(\alpha)} \int_{t_{0}}^{t}(t-s)^{\alpha-1} T(t-s) f(s, u(s), G u(s), S u(s)) d s \\
+\frac{1}{\Gamma(\alpha)} \int_{t_{0}}^{t}(t-s)^{\alpha-1} T(t-s)\left(A-A^{\alpha}\right) u(s) d s .
\end{gathered}
$$

Consequently, we have the following.

Corollary 2.2 If $\in L^{1}\left(\left[t_{0}, T\right]: X\right)$ then for every $u_{0} \in X$ the initial value problem (1.1) has at most one solution. If it has a solution, the solution is given by (2.1).

For every $f \in L^{1}\left(\left[t_{0}, T\right]: X\right)$ the right-hand side of Eq. (2.1) is a continuous function on $\left[t_{0} ; T\right]$. It is natural to consider it as a general solution of (1.1) even if it is not differentiable of order $\alpha$ and does not strictly satisfy the equation in the sense of Definition 2.1; we therefore define the following.

Definition 2.3 Let $A$ be infinitesimal generator of a $C_{0}$-semigroup $T(t)$. Let $u_{0} \in X$ and $f \in L^{1}\left(\left[t_{0}, T\right]: X\right)$. The function $u \in C\left(\left[t_{0}, T\right],: X\right)$ is given by

$$
\begin{aligned}
u(t)= & T\left(t-t_{0}\right) u_{0}+\frac{1}{\Gamma(\alpha)} \int_{t_{0}}^{t}(t-s)^{\alpha-1} T(t-s) f(s, u(s), G u(s), S u(s)) d s \\
& +\frac{1}{\Gamma(\alpha)} \int_{t_{0}}^{t}(t-s)^{\alpha-1} T(t-s)\left(A-A^{\alpha}\right) u(s) d s, \quad t_{0} \leq t \leq T,
\end{aligned}
$$

is the mild solution of the initial value problem (1.1) on $\left[t_{0}, T\right]$. 
By analogy to the developments [2-4, 6, 14], we can see that the function $u \in C\left(\left[t_{0}, T\right]\right.$ : $X)$ is called a mild solution of

$$
\begin{aligned}
& \left.\left.u^{(\alpha)}(t)=A u(t)+f(t), \quad t_{0}<t<T ; \alpha \in\right] 0,1\right], \\
& u\left(t_{0}\right)=u_{0}
\end{aligned}
$$

if

$$
u(t)=T\left(t-t_{0}\right) u_{0}+\frac{1}{\Gamma(\alpha)} \int_{t_{0}}^{t}(t-s)^{\alpha-1} T(t-s) f(s) d s, \quad t_{0} \leq t \leq T .
$$

However, it is easy to see that this concept of a solution is not realistic. In fact, if $A^{\alpha}=A$ and $f(t) \equiv f(t, u(t), G u(t), S u(t))$, the two definitions of a mild solution given by (2.2) and (2.4) coincide. Throughout this paper, we assume that the linear operator A satisfies the identity $A^{\alpha}=A$.

Definition 2.4 We say that $f:\left[t_{0}, T\right] \times X \times X \times X \rightarrow X$ is Lipschitz continuous if there is a constant $C$ such that

$$
\left\|f\left(t_{1}, x_{1}, y_{1}, z_{1}\right)-f\left(t_{2}, x_{2}, y_{2}, z_{2}\right)\right\| \leq C\left(\left|t_{1}-t_{2}\right|+\left\|x_{1}-x_{2}\right\|+\left\|y_{1}-y_{2}\right\|+\left\|z_{1}-z_{2}\right\|\right),
$$

where $t_{1}, t_{2} \in\left[t_{0}, T\right]$.

To prove our main results we need the following.

Theorem 2.5 ([6]) Let $f:\left[t_{0}, T\right] \times X \times X \times X \rightarrow X$ be continuous in $t$ on $\left[t_{0}, T\right]$ and uniformly Lipschitz continuous (with constant $L$ ) on $X$. If A is the generator of a strongly continuous semigroup $\{T(t) ; t \geq 0\}$ on a Banach space $X$, then for every $u_{0} \in X$ the initial value problem (1.1) has a unique mild solution $u \in C\left(\left[t_{0}, T\right]: X\right)$.

As a consequence of Theorem 2.5 we have the following.

Corollary 2.6 If $A$ and $f$ satisfy the conditions of Theorem 2.5 then for every $g \in C\left(\left[t_{0}, T\right]\right.$ : $X)$ the integral equation

$$
w(t)=g(t)+\frac{1}{\Gamma(\alpha)} \int_{t_{0}}^{t}(t-s)^{\alpha-1} T(t-s) f(s, w(s), G w(s), S w(s)) d s
$$

has a unique solution $w \in C\left(\left[t_{0}, T\right]: X\right)$.

Theorem 2.7 Let $A$ be the infinitesimal generator of a $C_{0}$ semigroup $T(t)$, let $f \in L^{1}\left(\left[t_{0}, T\right]\right.$ : $X)$ be continuous on $\left.] t_{0}, T\right]$ and

$$
v(t)=\frac{1}{\Gamma(\alpha)} \int_{t_{0}}^{t}(t-s)^{\alpha-1} T(t-s) f(s, u(s), G u(s), S u(s)) d s .
$$

The initial value problem (1.1) has a solution $u$ on $\left[t_{0}, T\left[\right.\right.$ for every $u_{0} \in D(A)$ if one of the following conditions is satisfied: 
(1) $v(t)$ is continuously differentiable of order $\alpha$ on $] t_{0}, T[$.

(2) $v(t) \in D(A)$ for $t_{0}<t<T$ and $A v(t)$ is continuous on $] t_{0}, T[$.

If (1.1) has a solution $u$ on $\left[t_{0}, T\left[\right.\right.$ for some $u_{0} \in D(A)$ then $v(t)$ satisfies both (1) and (2).

Proof If the initial value problem (1.1) has a solution $\mathrm{u}$ for some $u_{0} \in D(A)$ then this solution is given by (2.4). Consequently, $v(t)=u(t)-T\left(t-t_{0}\right) u_{0}$ is differentiable of order $\alpha$ for $t>t_{0}$ as the difference of two such differentiable functions of order $\alpha$ and $v^{(\alpha)}(t)=u^{(\alpha)}(t)-A T\left(t-t_{0}\right) u_{0}$ is obviously continuous on $] t_{0}, T$ [. Therefore, (1) is satisfied. Also, if $u_{0} \in D(A), T\left(t-t_{0}\right) u_{0} \in D(A)$ for $t>t_{0}$ and therefore $v(t)=u(t)-T\left(t-t_{0}\right) u_{0} \in D(A)$ for $t>t_{0}$ and $A v(t)=A u(t)-A T\left(t-t_{0}\right) u_{0}=u^{(\alpha)}(t)-f(t, u(t), G u(t), S u(t))-A T\left(t-t_{0}\right) u_{0}$ is continuous on $] t_{0}, T[$. Thus also (2) is satisfied.

On the other hand, it is easy to verify that for $h>0$ the identity

$$
\begin{aligned}
\frac{T(h)-I}{h} v(t)= & \frac{v(t+h)-v(t)}{h} \\
& -\frac{1}{h \Gamma(\alpha)} \int_{t}^{t+h}(t+h-s)^{\alpha-1} T(t+h-s) f(s, \ldots) d s
\end{aligned}
$$

holds and from the continuity of $f$ it is clear that the second term of the right-hand side of (2.6) has the limit $f(t, \ldots)$ as $h \rightarrow 0$. If $v(t)$ is continuously differentiable of order $\alpha$ on ] $t_{0}, T$ [ then it follows from (2.6) that $v(t) \in D(A)$ for $t_{0}<t<T$ and $A v(t)=v^{(\alpha)}-f(t, \ldots)$. Since $v(0)=0$ it follows that $u(t)=T\left(t-t_{0}\right) u_{0}+v(t)$ is the solution of the initial value problem (1.1) for $u_{0} \in D(A)$. If $v(t) \in D(A)$ it follows from (2.6) that $v(t)$ is differentiable of order $\alpha$ from the right at $t$ and the right $\alpha$-derivative $D_{+}^{(\alpha)} v(t)$ of $v$ satisfies $D_{+}^{(\alpha)} v(t)=$ $A v(t)+f(t, \ldots)$. Since $D_{+}^{(\alpha)} v(t)$ is continuous, $v(t)$ is continuously differentiable of order $\alpha$ and $v^{(\alpha)}(t)=A v(t)+f(t, \ldots)$. Since $v(0)=0, u(t)=T\left(t-t_{0}\right) u_{0}+v(t)$ is the solution of the (1.1) for $u_{0} \in D(A)$ and the proof is complete.

From Theorem 2.7, we have the following corollary.

Corollary 2.8 Let $A$ be the infinitesimal generator of a $C_{0}$-semigroup $T(t)$. Iff is continuously differentiable of order $\alpha$ on $\left[t_{0}, T\right]$ then the initial value problem (1.1) has a solution $u$ on $\left[t_{0}, T\left[\right.\right.$ for every $u_{0} \in D(A)$.

Proof We have

$$
\begin{aligned}
v(t)= & \frac{1}{\Gamma(\alpha)} \int_{t_{0}}^{t}(t-s)^{\alpha-1} T(t-s) f(s, u(s), G u(s), S u(s)) d s \\
& +\frac{1}{\Gamma(\alpha)} \int_{t_{0}}^{t} s^{\alpha-1} T(s) f(t-s, u(t-s), G u(t-s), S u(t-s)) d s .
\end{aligned}
$$

It is clear from (2.7) that $v(t)$ is differentiable of order $\alpha$ for $t>t_{0}$ and that the $\alpha$-derivative,

$$
\begin{aligned}
v^{(\alpha)}(t)= & T(t) f(0, u(0) G u(0), S u(0)) \\
& +\frac{1}{\Gamma(\alpha)} \int_{t_{0}}^{t} s^{\alpha-1} T(s) f(t-s, u(t-s), G u(t-s), S u(t-s)) d s
\end{aligned}
$$




$$
\begin{aligned}
= & T(t) f(0, u(0), G u(0), S u(0)) \\
& +\frac{1}{\Gamma(\alpha)} \int_{t_{0}}^{t}(t-s)^{\alpha-1} T(t-s) D^{\alpha} f(s, u(s), G u(s), S u(s)) d s,
\end{aligned}
$$

is continuous on $] t_{0}, T[$. The result therefore follows now from Theorem 2.7(1).

Theorem 2.9 Let $A$ be the infinitesimal generator of a $C_{0}$-semigroup $T(t)$ on $X$. If $f$ : $\left[t_{0}, T\right] \times X \times X \times X \rightarrow X$ is continuously differentiable from $\left[t_{0}, T\right] \times X \times X \times X$ into $X$ then the mild solution of $(1.1)$ with $u_{0} \in D(A)$ is a classical solution of the initial value problem.

Proof We note first that the continuous differentiability of $f$ from $\left[t_{0}, T\right] \times X \times X \times X$ into $X$ implies that $f$ is continuous in $t$ and Lipschitz continuous in $u$, uniformly in $t$ on $\left[t_{0}, T\right]$. Therefore the initial value problem (1.1) possesses a unique mild solution $u$ on $\left[t_{0}, T\right]$ by Theorem 2.5. Next we show that this mild solution is continuously differentiable on $\left[t_{0}, T\right]$. To this end we set $B(s)=\frac{\partial}{\partial u} f(s, u, G u, S u)$ and

$$
\begin{aligned}
g(t)= & T\left(t-t_{0}\right) f\left(t_{0}, u\left(t_{0}\right), G u\left(t_{0}\right), S u\left(t_{0}\right)\right)-A T\left(t-t_{0}\right) u_{0} \\
& +\frac{1}{\Gamma(\alpha)} \int_{t_{0}}^{t}(t-s)^{\alpha-1} T(t-s) \frac{\partial}{\partial s} f(s, u, G u, S u) d s .
\end{aligned}
$$

From our assumption it follows that $g(t) \in C\left(\left[t_{0}, T\right]: X\right)$ and that the function $h(t, u, G u$, $S u)=B(t) E(u, G u, S u)$ is continuous in $t$ from $\left[t_{0}, T\right]$ into $X$ and uniformly Lipschitz continuous in $u$ since $s \rightarrow B(s)$ is continuous from $\left[t_{0}, T\right]$ into $B(X)$. Let $w$ be the solution of the integral equation

$$
w(t)=g(t)+\frac{1}{\Gamma(\alpha)} \int_{t_{0}}^{t}(t-s)^{\alpha-1} T(t-s) B(s) w(s) d s .
$$

The existence and uniqueness of $w(t) \in C\left(\left[t_{0}, T\right]: X\right)$ follow from Corollary 2.6. Moreover, from our assumptions we have

$$
\begin{aligned}
& f(s, u(s+h), G u(s+h), S u(s+h))-f(s, u(s), G u(s), S u(s)) \\
& \quad=B(s)(u(s+h)-u(s))+w_{1}(s, h)
\end{aligned}
$$

and

$$
\begin{aligned}
f(s+h, u(s+h), G u(s+h), S u(s+h))-f(s, u(s+h), G u(s+h), S u(s+h)) \\
\quad=\frac{\partial}{\partial s} f(s, u(s+h), G u(s+h), S u(s+h)) \cdot h+w_{2}(s, h)
\end{aligned}
$$

where $h^{-1}\left\|w_{i}(s, h)\right\| \rightarrow 0$ as $h \rightarrow 0$ uniformly on $\left[t_{0}, T\right]$ for $i=1,2$.

Adding (2.10) and (2.11) we have

$$
\begin{aligned}
& f(s+h, u(s+h), G u(s+h), S u(s+h))-f(s, u(s), G u(s), S u(s)) \\
&= B(s)(u(s+h)-u(s))+w_{1}(s, h) \\
&+\frac{\partial}{\partial s} f(s, u(s+h), G u(s+h), S u(s+h)) \cdot h+w_{2}(s, h) .
\end{aligned}
$$


If $w_{h}(t)=h^{-1}(u(t+h)-u(t))-w(t)$ then from the definition of $u$ and (2.9) we obtain

$$
\begin{aligned}
& w_{h}(t)=h^{-1}[u(t+h)-u(t)]-w(t) \\
& =h^{-1}\left[T\left(t+h-t_{0}\right) u_{0}-T\left(t-t_{0}\right) u_{0}\right. \\
& +\frac{1}{\Gamma(\alpha)} \int_{t_{0}}^{t+h}(t+h-s)^{\alpha-1} T(t+h-s) f(s, u(s), G u(s), S u(s)) d s \\
& \left.-\frac{1}{\Gamma(\alpha)} \int_{t_{0}}^{t} T(t-s)(t-s)^{\alpha-1} f(s, u(s), G u(s), S u(s)) d s\right] \\
& -T\left(t-t_{0}\right) f\left(t_{0}, u\left(t_{0}\right), G u\left(t_{0}\right), S u\left(t_{0}\right)\right)+A T\left(t-t_{0}\right) u_{0} \\
& -\frac{1}{\Gamma(\alpha)} \int_{t_{0}}^{t}(t-s)^{\alpha-1} T(t-s) \frac{\partial}{\partial s} f(s, u(s), G u(s), S u(s)) d s \\
& -\frac{1}{\Gamma(\alpha)} \int_{t_{0}}^{t}(t-s)^{\alpha-1} T(t-s) B(s) w(s) d s, \\
& w_{h}(t)=h^{-1}[u(t+h)-u(t)]-w(t) \\
& =h^{-1}\left[T\left(t+h-t_{0}\right) u_{0}-T\left(t-t_{0}\right) u_{0}\right. \\
& +\frac{1}{\Gamma(\alpha)} \int_{t_{0}}^{t+h}(t+h-s)^{\alpha-1} T(t+h-s) f(s, u(s), G u(s), S u(s)) d s \\
& +\frac{1}{\Gamma(\alpha)} \int_{t_{0}+h}^{t+h} T(t-s)(t-s)^{\alpha-1} f(s, u(s), G u(s), S u(s)) d s \\
& \left.-\frac{1}{\Gamma(\alpha)} \int_{t_{0}}^{t} T(t-s)(t-s)^{\alpha-1} f(s, u(s), G u(s), S u(s)) d s\right] \\
& -T\left(t-t_{0}\right) f\left(t_{0}, u\left(t_{0}\right), G u\left(t_{0}\right), S u\left(t_{0}\right)\right)+A T\left(t-t_{0}\right) u_{0} \\
& -\frac{1}{\Gamma(\alpha)} \int_{t_{0}}^{t}(t-s)^{\alpha-1} T(t-s) \frac{\partial}{\partial s} f(s, u(s), G u(s), S u(s)) d s \\
& -\frac{1}{\Gamma(\alpha)} \int_{t_{0}}^{t}(t-s)^{\alpha-1} T(t-s) B(s) w(s) d s \\
& w_{h}(t)=h^{-1}\left[T\left(t+h-t_{0}\right) u_{0}-T\left(t-t_{0}\right) u_{0}\right. \\
& +\frac{1}{\Gamma(\alpha)} \int_{t_{0}}^{t_{0}+h}(t+h-s)^{\alpha-1} T(t+h-s) f(s, u(s), G u(s), S u(s)) d s \\
& +\frac{1}{\Gamma(\alpha)} \int_{t_{0}}^{t} T(t-s)(t-s)^{\alpha-1}(f(s+h, u(s+h), G u(s+h), S u(s+h)) \\
& -f(s, u(s), G u(s), S u(s))) d s] \\
& -T\left(t-t_{0}\right) f\left(t_{0}, u\left(t_{0}\right), G u\left(t_{0}\right), S u\left(t_{0}\right)\right)+A T\left(t-t_{0}\right) u_{0} \\
& -\frac{1}{\Gamma(\alpha)} \int_{t_{0}}^{t}(t-s)^{\alpha-1} T(t-s) \frac{\partial}{\partial s} f(s, u(s), G u(s), S u(s)) d s \\
& -\frac{1}{\Gamma(\alpha)} \int_{t_{0}}^{t}(t-s)^{\alpha-1} T(t-s) B(s) w(s) d s .
\end{aligned}
$$


Now, from (2.10) and (2.11), we have

$$
\begin{aligned}
w_{h}(t)= & {\left[h^{-1}\left(T\left(t+h-t_{0}\right) u_{0}-T\left(t-t_{0}\right) u_{0}\right)+A T\left(t-t_{0}\right) u_{0}\right] } \\
& +\frac{1}{h \Gamma(\alpha)} \int_{t_{0}}^{t_{0}+h}(t-s)^{\alpha-1} T(t+h-s) f(s, u(s), G u(s), S u(s)) d s \\
& -T\left(t-t_{0}\right) f\left(t_{0}, u\left(t_{0}\right), G u\left(t_{0}\right), S u\left(t_{0}\right)\right) \\
& +\frac{1}{h \Gamma(\alpha)} \int_{t_{0}}^{t} T(t-s)(t-s)^{\alpha-1}\left[h \times\left(B(s)(u(s+h)-u(s))+w_{1}(s, h)\right.\right. \\
& \left.\left.\left.+\frac{\partial}{\partial s} f(s, u(s+h), G u(s+h), S u(s+h))\right)+w_{2}(s, h)\right)\right] d s \\
& -\frac{1}{\Gamma(\alpha)} \int_{t_{0}}^{t}(t-s)^{\alpha-1} T(t-s) \frac{\partial}{\partial s} f(s, u(s), G u(s), S u(s)) d s \\
& -\frac{1}{\Gamma(\alpha)} \int_{t_{0}}^{t}(t-s)^{\alpha-1} T(t-s) B(s) w(s) d s
\end{aligned}
$$

by rearrangement of the terms of (2.15) we have

$$
\begin{aligned}
w_{h}(t)= & {\left[h^{-1}\left(T\left(t+h-t_{0}\right) u_{0}-T\left(t-t_{0}\right) u_{0}\right)+A T\left(t-t_{0}\right) u_{0}\right] } \\
& +\frac{1}{h \Gamma(\alpha)} \int_{t_{0}}^{t_{0}+h}(t-s)^{\alpha-1} T(t+h-s) f(s, u(s), G u(s), S u(s)) d s \\
& -T\left(t-t_{0}\right) f\left(t_{0}, u\left(t_{0}\right), G u\left(t_{0}\right), S u\left(t_{0}\right)\right) \\
& \frac{1}{\Gamma(\alpha)} \int_{t_{0}}^{t} T(t-s)(t-s)^{\alpha-1} B(s)[(u(s+h)-u(s))-w(s)] d s \\
& +\frac{1}{\Gamma(h \alpha)} \int_{t_{0}}^{t} T(t-s)(t-s)^{\alpha-1}\left[w_{1}(s, h)+w_{2}(s, h)\right] d s \\
& +\frac{1}{\Gamma(\alpha)} \int_{t_{0}}^{t} T(t-s)(t-s)^{\alpha-1}\left[\frac{\partial}{\partial s} f(s, u(s+h), G u(s+h), S u(s+h))\right. \\
& \left.-\frac{\partial}{\partial s} f(s, u(s), G u(s), S u(s))\right] d s .
\end{aligned}
$$

Now

$$
\begin{aligned}
w_{h}(t)= & {\left[h^{-1}\left(T\left(t+h-t_{0}\right) u_{0}-T\left(t-t_{0}\right) u_{0}\right)+A T\left(t-t_{0}\right) u_{0}\right] } \\
& +\frac{1}{h \Gamma(\alpha)} \int_{t_{0}}^{t}(t-s)^{\alpha-1} T(t-s)\left(w_{1}(s, h)+w_{2}(s, h)\right) d s \\
& +\frac{1}{\Gamma(\alpha)} \int_{t_{0}}^{t}(t-s)^{\alpha-1} T(t-s)\left(\frac{\partial}{\partial s} f(s, u(s+h), G u(s+h), S u(s+h))\right. \\
& -\frac{\partial}{\partial s} f(s, u(s), G u(s), S u(s)) d s \\
& +\frac{1}{h \Gamma(\alpha)} \int_{t_{0}}^{t_{0}+h}(t+h-s)^{\alpha-1} T(t+h-s) f(s, u(s), G u(s), S u(s)) d s \\
& -T\left(t-t_{0}\right) f\left(t_{0}, u\left(t_{0}\right), G u\left(t_{0}\right), S u\left(t_{0}\right)\right)+\frac{1}{\Gamma(\alpha)} \int_{t_{0}}^{t}(t-s)^{\alpha-1} T(t-s) w_{h}(t) d s .
\end{aligned}
$$


Now

$$
\begin{aligned}
\left\|w_{h}(t)\right\| \leq & \left\|\left[h^{-1}\left(T\left(t+h-t_{0}\right) u_{0}-T\left(t-t_{0}\right) u_{0}\right)+A T\left(t-t_{0}\right) u_{0}\right]\right\| \\
& +h^{-1}\left\|\frac{1}{\Gamma(\alpha)} \int_{t_{0}}^{t}(t-s)^{\alpha-1} T(t-s)\left(w_{1}(s, h)+w_{2}(s, h)\right) d s\right\| \\
& +\| \frac{1}{\Gamma(\alpha)} \int_{t_{0}}^{t}(t-s)^{\alpha-1} T(t-s)\left(\frac{\partial}{\partial s} f(s, u(s+h), G u(s+h), S u(s+h))\right. \\
& -\frac{\partial}{\partial s} f(s, u(s), G u(s), S u(s)) d s \| \\
& +h^{-1} \| \frac{1}{\Gamma(\alpha)} \int_{t_{0}}^{t_{0}+h}(t+h-s)^{\alpha-1} T(t+h-s) f(s, u(s), G u(s), S u(s)) d s \\
& -T\left(t-t_{0}\right) f\left(t_{0}, u\left(t_{0}\right), G u\left(t_{0}\right), S u\left(t_{0}\right)\right) \| \\
& +\left\|\frac{1}{\Gamma(\alpha)} \int_{t_{0}}^{t}(t-s)^{\alpha-1} T(t-s) w_{h}(t) d s\right\| .
\end{aligned}
$$

The norm of each one of the four first terms on the right-hand side of (2.16) tends to zero as $h \rightarrow 0$. Therefore we have

$$
\left\|w_{h}(t)\right\| \leq \rho(h)+\frac{M}{\Gamma(\alpha)} \int_{t_{0}}^{t}(t-s)^{\alpha-1}\left\|w_{h}(s)\right\| d s
$$

where $M=\max \left\{\|T(t-s)\|\|B(s)\|: t_{0} \leq s \leq T\right\}$ and $\rho(h) \rightarrow 0$ as $h \rightarrow 0$. From (2.17) it follows by the generalized Gronwall inequality [5] that

$$
\begin{aligned}
\left\|w_{h}(t)\right\| & \leq \rho(h)+\int_{t_{0}}^{t}\left[\sum_{n=1}^{\infty} \frac{M^{n}}{\Gamma(n \alpha)}(t-s)^{n \alpha-1} \rho(h) d s\right] \\
& =\rho(h) \sum_{n=0}^{\infty} \frac{M^{n}\left(t-t_{0}\right)^{n \alpha}}{\Gamma(n \alpha+1)} \\
& =\rho(h) E_{\alpha}\left(M\left(t-t_{0}\right)^{\alpha}\right), \quad \text { where } E_{\alpha}(z)=\sum_{k=0}^{\infty} \frac{z^{k}}{\Gamma(k \alpha+1)}
\end{aligned}
$$

and therefore $\left\|w_{h}(t)\right\| \rightarrow 0$ as $h \rightarrow 0$. This implies that $u(t)$ is differentiable on $\left[t_{0}, T\right]$.

Finally, to show $u$ is the classical solution of (1.1) we note that from the continuous differentiability of $u$ and the assumption of the differentiability of $f$ it follows that $s \rightarrow$ $f(s, u(s), G u(s), S u(s))$ is continuously differentiable on $\left[t_{0}, T\right]$. From Corollary 2.8 it then follows that

$$
v(t)=T\left(t-t_{0}\right) u_{0}+\frac{1}{\Gamma(\alpha)} \int_{t_{0}}^{t}(t-s)^{\alpha-1} T(t-s) f(s, u(s), G u(s), S u(s)) d s
$$

is the classical solution of the initial value problem

$$
\begin{aligned}
& v^{(\alpha)}(t)=A v(t)+f(t, u(t), G u(t), S u(t)), \quad t>t_{0}, \alpha \in(0,1], \\
& v\left(t_{0}\right)=u_{0} .
\end{aligned}
$$


But, by definition, $u$ is a mild solution of (2.16) and by the uniqueness of the mild solution of (2.16) it follows that $u=v$. Thus, $u$ is a classical solution of the initial value problem (1.1).

Definition 2.10 A function $u$ which is differential of order $\alpha$ almost everywhere on $\left[t_{0}, T\right]$ such that $u^{(\alpha)} \in C^{(\alpha)}\left(\left[t_{0}, T\right]: X\right)$ is called a strong solution of the initial value problem (1.1) if

(1) $u^{(\alpha)}(t)=A u(t)+f\left(t, u(t), G u(t), S u(t), t_{0}<t<T, 0<\alpha \leq 1\right.$ almost everywhere on $\left[t_{0}, T\right]$.

(2) $u\left(t_{0}\right)=u_{0}$.

With the same proof as of Theorem 2.7 we have the following.

Theorem 2.11 Let $A$ be the infinitesimal generator of a $C_{0}$ semigroup $T(t)$, let $f \in$ $L^{1}\left(\left[t_{0}, T\right]: X\right)$ be continuous on $\left.] t_{0}, T\right]$ and

$$
v(t)=\frac{1}{\Gamma(\alpha)} \int_{t_{0}}^{t}(t-s)^{\alpha-1} T(t-s) f\left(s, u(s), G u(s), S u(s) d s, \quad t \in\left[t_{0}, T\right] .\right.
$$

The initial value problem (1.1) has a strong solution $u$ on $\left[t_{0}, T\right]$ for every $u_{0} \in D(A)$ if one of the following conditions is satisfied:

(1) $v(t)$ is differentiable of order $\alpha$ on $\left[t_{0}, T\right]$ and $v^{(\alpha)} \in C^{(\alpha)}\left(\left[t_{0}, T\right]: X\right)$.

(2) $v(t) \in D(A)$ a.e. on $\left[t_{0}, T\right]$ and $A v(t) \in L^{1}\left(\left[t_{0}, T\right]: X\right)$.

If (1.1) has a strong solution $u$ on $\left[t_{0}, T\right]$ for some $u_{0} \in D(A)$ then $v$ satisfies both (1) and (2).

As a consequence of Theorem 2.11 we have the following.

Corollary 2.12 Let $X$ be a reflexive Banach space and let $A$ be the infinitesimal generator of a $C_{0}$-semigroup $T(t)$ on $X$. Iff is Lipschitz continuous on $\left[t_{0}, T\right]$ then for each $u_{0} \in D(A)$ the initial value problem (1.1) has a unique strong solution on $\left[t_{0}, T\right]$ given by

$$
u(t)=T\left(t-t_{0}\right) u_{0}+\frac{1}{\Gamma(\alpha)} \int_{t_{0}}^{t}(t-s)^{\alpha-1} T(t-s) f(s, \ldots) d s, \quad t \in\left[t_{0}, T\right] .
$$

To prove that the mild solution of the initial value problem (1.1) is a strong solution, we need the following lemma.

Lemma 2.13 ([13]) For all $\alpha>0$ and $\beta>-1$, we have

$$
\int_{0}^{t}(t-s)^{\alpha-1} s^{\beta} d s=\frac{t^{\alpha+\beta} \Gamma(\alpha) \Gamma(\beta+1)}{\Gamma(\alpha+\beta+1)} .
$$

Theorem 2.14 Let $A$ be the infinitesimal generator of a $C_{0}$-semigroup $T(t)$ on a reflexive Banach space $X$. If $f:\left[t_{0}, T\right] \times X \times X \times X \rightarrow X$ is Lipschitz continuous in all variables, $u_{0} \in D(A)$ and $u$ is the mild solution of the initial value problem (1.1) then $u$ is the strong solution of this initial value problem (1.1). 
Proof Let $\|T(t)\| \leq M$ and $\|f(t, u(t), G u(t), S u(t))\| \leq N$ for $t_{0} \leq t \leq T$ and let $f$ satisfy the Lipschitz condition. For $0<h<t-t_{0}$ we have

$$
\begin{aligned}
u(t+h)-u(t)= & T\left(t+h-t_{0}\right) u_{0}-T\left(t-t_{0}\right) u_{0} \\
& +\frac{1}{\Gamma(\alpha)} \int_{t_{0}}^{t_{0}+h}(t-s)^{\alpha-1} T(t+h-s) f(s, u(s), G u(s), S u(s)) d s \\
& +\frac{1}{\Gamma(\alpha)} \int_{t_{0}}^{t}(t-s)^{\alpha-1} T(t-s)[f(s+h, u(s+h), G u(s+h), S u(s+h)) \\
& -f(s, u(s), G u(s), S u(s))] d s
\end{aligned}
$$

and therefore, by using Lemma 2.13 , we have

$$
\begin{aligned}
&\|u(t+h)-u(t)\| \\
& \leq h M\left\|A u_{0}\right\|+h M N C_{1} \\
&+\frac{1}{\Gamma(\alpha)} \int_{t_{0}}^{t}\left\|(t-s)^{\alpha-1}\right\|\|T(t-s)\| \\
& \quad[\|f(s+h, u(s+h), G u(s+h), S u(s+h))-f(s, u(s), G u(s), S u(s))\|] d s \\
& \leq h M\left\|A u_{0}\right\|+h M N C_{1}+\frac{M}{\Gamma(\alpha)} \int_{t_{0}}^{t}\left\|(t-s)^{\alpha-1}\right\| c[h+\|u(s+h)-u(s)\| \\
&+\|(G u(s+h)-G u(s))\|+\|S u(s+h)-S u(s)\|] d s,
\end{aligned}
$$

where $C_{1}=\frac{1}{\Gamma(\alpha+1)}$. But

$$
\begin{aligned}
& \frac{M}{\Gamma(\alpha)} \int_{t_{0}}^{t}\left\|(t-s)^{\alpha-1}\right\|\|(G u(s+h)-G u(s))\| d s \\
& \quad \leq \frac{M}{\Gamma(\alpha)} \int_{t_{0}}^{t}\left\|(t-s)^{\alpha-1}\right\| \int_{t_{0}}^{s}|G(s, \tau)|\|u(s+h)-u(s)\| d \tau d s \\
& \quad \leq \frac{M}{\Gamma(\alpha)} \int_{t_{0}}^{t}\left\|(t-s)^{(\alpha-1)}\right\|\|G\|\|u(s+h)-u(s)\| d s \\
& \quad \leq \frac{M\|G\|}{\Gamma(\alpha)} \int_{t_{0}}^{t}\left\|(t-s)^{(\alpha-1)}\right\|\|u(s+h)-u(s)\| d s \\
& \quad \leq \frac{M\|G\|}{\Gamma(\alpha)} \int_{t_{0}}^{t}(t-s)^{\alpha-1}\|u(s+h)-u(s)\| d s
\end{aligned}
$$

similarly

$$
\begin{aligned}
& \frac{M}{\Gamma(\alpha)} \int_{t_{0}}^{t}\left\|(t-s)^{\alpha-1}\right\|\|(S u(s+h)-S u(s))\| d s \\
& \quad \leq \frac{M}{\Gamma(\alpha)} \int_{t_{0}}^{t}\left\|(t-s)^{\alpha-1}\right\| \int_{t_{0}}^{s}|K(s, \tau)|\|u(s+h)-u(s)\| d \tau d s \\
& \quad \leq \frac{M}{\Gamma(\alpha)} \int_{t_{0}}^{t}\left\|(t-s)^{(\alpha-1)}\right\|\|S\|\|u(s+h)-u(s)\| d s
\end{aligned}
$$




$$
\begin{aligned}
& \leq \frac{M\|S\|}{\Gamma(\alpha)} \int_{t_{0}}^{t}\left\|(t-s)^{(\alpha-1)}\right\|\|u(s+h)-u(s)\| d s \\
& \leq \frac{M\|S\|}{\Gamma(\alpha)} \int_{t_{0}}^{t}(t-s)^{\alpha-1}\|u(s+h)-u(s)\| d s,
\end{aligned}
$$

then we have

$$
\begin{aligned}
\|u(t+h)-u(t)\| \leq & h M\left\|A u_{0}\right\|+h M N C_{1} \\
& +\frac{M h(1+\|G\|+\|S\|)}{\Gamma(\alpha)} \int_{t_{0}}^{t}(t-s)^{\alpha-1}\|u(s+h)-u(s)\| d s
\end{aligned}
$$

so that

$$
\begin{aligned}
& \|u(t+h)-u(t)\| \\
& \quad \leq k_{1} h+\frac{M h}{\Gamma(\alpha)} \int_{t_{0}}^{t}(t-s)^{\alpha-1}\|u(s+h)-u(s)\| d s, \quad \text { where } k_{1}=M\left\|A u_{0}\right\|+M N C_{1},
\end{aligned}
$$

which by the generalized Gronwall inequality [5] implies

$$
\begin{aligned}
\|u(t+h)-u(t)\| & \leq k_{1} h+\int_{t_{0}}^{t} \sum_{n=1}^{\infty} \frac{(M h)^{n}}{\Gamma(n \alpha)}(t-s)^{n \alpha-1} k_{1} h d s \\
& =k_{1} h \sum_{n=1}^{\infty} \frac{(M h)^{n}\left(t-t_{0}\right)^{n \alpha}}{\Gamma(n \alpha+1)} \\
& =k_{1} h E_{\alpha}\left(M h\left(t-t_{0}\right)^{\alpha}\right), \quad \text { where } E_{\alpha}(z)=\sum_{k=0}^{\infty} \frac{z^{k}}{\Gamma(k \alpha+1)}
\end{aligned}
$$

and $u$ is Lipschitz continuous.

The Lipschitz continuity of $u$ combined with the Lipschitz continuity of $f$ implies that $t \rightarrow f(t, u(t), G u(t), S u(t))$ is Lipschitz continuous on $\left[t_{0}, T\right]$. From Corollary (2.12) it then follows that the initial value problem

$$
\begin{aligned}
& v^{(\alpha)}(t)=A \nu(t)+f(t, u(t), G u(t), S u(t)), \quad t>t_{0}, \alpha \in(0,1], \\
& v\left(t_{0}\right)=u_{0},
\end{aligned}
$$

has a unique strong solution $v$ on $\left[t_{0}, T\right]$ satisfying

$$
\begin{aligned}
v(t) & =T\left(t-t_{0}\right) u_{0}+\frac{1}{\Gamma(\alpha)} \int_{t_{0}}^{t}(t-s)^{\alpha-1} T(t-s) f(s, u(s), G u(s), S u(s)) d s \\
& =u(t)
\end{aligned}
$$

and so $u$ is a strong solution of (1.1).

\section{Conclusions}

In this paper, we investigated the existence and uniqueness of classical and strong solutions of a fractional semilinear evolution equation using the method of a semigroup and the Banach fixed theorem. 
Acknowledgements

The authors would like to thank the referees for useful comments and suggestions.

\section{Funding}

Not applicable.

\section{Availability of data and materials}

Not applicable.

\section{Competing interests}

The authors declare that there is no conflict of interest regarding the publication of this article.

\section{Authors' contributions}

Each author equally contributed to this paper, and read and approved the final manuscript.

\section{Author details}

${ }^{1}$ Department of Mathematics, Faculty of Sciences, Al Al-Bayt University, Mafraq, Jordan. ${ }^{2}$ Mathematics Department, Faculty of Applied Sciences, Umm Al-Qura University, Makkah, Saudi Arabia.

\section{Publisher's Note}

Springer Nature remains neutral with regard to jurisdictional claims in published maps and institutional affiliations.

Received: 16 May 2018 Accepted: 29 August 2018 Published online: 19 October 2018

\section{References}

1. Agarwal, R.P., Benchohra, M., Hamani, S.A.: Survey on existence results for boundary value problems of nonlinear fractional differential equations and inclusions. Acta Appl. Math. 109, 973-1033 (2010)

2. Balachandran, K., llamaran, S.: Existence and uniqueness of mild and strong solutions of a semilinear Evolution equation with nonlocal conditions. Indian J. Pure Appl. Math. 25(4), 411-418 (1994)

3. Belmekki, M., Benchohra, M.: Existence results for fractional order semilinear functional difierential with nondense domain. Nonlinear Anal. 72, 925-932 (2010)

4. Chang, Y.K., Kavitha, V., Arjunan, M.M.: Existence and uniqueness of mild solutions to a semi-linear integrodifferential equation of fractional order. Nonlinear Anal. 71, 5551-5559 (2009)

5. Haiping, Y., Jianming, G., Yongsheng, D.: A generalized Gronwall inequality and its application to a fractional differential equation. J. Math. Anal. Appl. 328, 1075-1081 (2007)

6. Jaradat, O., Al-Omari, A., Momani, S.: Existence of the mild solution for fractional semilinear initial calue problems. Nonlinear Anal. 69, 3153-3159 (2008)

7. Kilbas, A.A., Srivastava, H.M., Trujillo, J..: Theory and Applications of Fractional Differential Equations. North-Holland Mathematics Studies, vol. 204. Elsevier, Amsterdam (2006)

8. Lakshmikantham, V., Leela, S., Vasundhara Devi, J.: Theory of Fractional Dynamic Systems. Cambridge Academic Publishers, Cambridge (2009)

9. Miller, K.S., Ross, B.: An Introduction to the Fractional Calculus and Differential Equations. Wiley, New York (1993)

10. Pazy, A.: Semigroup of Linear Operators and Applications to Partial Differential Equations. Springer, New York (1983)

11. Podlubny, l.: Fractional Differential Equations. Academic Press, San Diego (1999)

12. Rehonan, M., Khan, R.: A numerical method for solving boundary value problems for fractional differential equations. Appl. Math. Model. 36(3), 894-907 (2012)

13. Tatar, N.E.: An impulsive nonlinear singular version of the Gronwall-Bihari inequality. J. Inequal. Appl. 2006, 84561 (2006). https://doi.org/10.1155/JIA/2006/84561

14. Zhou, Y., Jiao, F: Nonlocal Cauchy problem for fractional evolution equations. Nonlinear Anal. 11, 4465-4475 (2010)

\section{Submit your manuscript to a SpringerOpen ${ }^{\circ}$ journal and benefit from:}

- Convenient online submission

- Rigorous peer review

- Open access: articles freely available online

- High visibility within the field

- Retaining the copyright to your article

Submit your next manuscript at $\boldsymbol{~ s p r i n g e r o p e n . c o m ~}$ 